\title{
MASSES, RADII, TEMPERATURES, LUMINOSITIES AND SURFACE COMPOSITIONS OF WHITE DWARFS
}

\author{
Jesse L. Greenstein \\ Hale Observatorles \\ California Institute of Technology
}

My title is so inclusive that it is not possible to find significant subject matter not in direct conflict with the specialists' talks scheduled throughout this Colloquium. I intend, however, to emphasize areas where it is probable that much remains to be done. From the Kiel group come printouts for a large family of models, first for hydrogen and recently for helium-dominated atmospheres, which $D$. Koester has kindly made avallable. Hydrogen atmospheres for a wide varlety of temperatures by Shipman and McGraw and Shipman exist also in computer printout form. Models of mixed He/H composition are less avallable, but are being prepared. Some hydrogen-deficient stars are the richest in metals, so that models for a varlety of metal/helium ratios will need to be computed, and synthetic spectra. Clearly, further models with differing $\mathrm{c} / \mathrm{He}$ ratios are needed. The successful observations in the ultraviolet with the IUE also demand computations of Lyman- $\alpha$ profiles using modern broadening theory, and a few now exist (Koester 1979). Present observational capabilities require quite detailed predicted fluxes and He II, He I line profiles.

The Kiel group (Koester, Schulz, and Weidemann 1979) and Shipman (1979), Shipman and collaborators (preprint) have used existing colors or my multichannel energy distributions to compute radil from luminosities and temperatures to obtain masses from the theoretical carboncore mass-radius relation. As these will be discussed, I need only say that radil for stars of known parallax, and masses, together with their variance, now exist. Shipman (1979) has discussed selection effects in a magnitude-limited sample. The most severely magnitudelimited sample, however, is that selection - the apparently brightest stars (most luminous, 1.e., lowest masses)-observed for gravitational redshift by Wegner, and by myself and collaborators. Yet a slight discrepancy now exists in that the observed mean redshift is slightly too large for the mean radius and mass otherwise derived. The mass, as derived from the radius, is sensitive to the effective temperature and appears to be most commonly in the range 0.5 to $0.7 \mathrm{M}$ for DA's. The distance, $d$, enters from $L \propto d^{2} f ; f$ is the integrated $f$ lux, determined if possible, from wide-range energy distributions. A monochromatic flux predicted from a model, $\mathrm{H}$, may be used, with less certainty. In either case, $R / d \propto T^{-2}$; fractional errors are $\Delta R / R=$ $-2 \Delta T / T+\Delta d / d$. The enormous improvement in the parallaxes made possible by the U. S. Naval Observatory program, initiated by Kaj Strand, must 
be gratefully acknowledged. But as an observer of extremes, I must still ask for more parallaxes of the hottest and intrinsically brightest

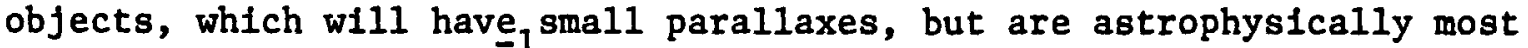
important. Since $M \approx R^{-1}$ over the middle of the known range of white dwarf masses, the predicted gravitational redshift, $M / R \propto 4 \Delta T / T-2 \Delta d / d$. Thus, the temperature and parallax, both poorly determined at the hot end, enter critically into the expected redshift.

I should mention that In single white dwarfs those few bright DA stars observed by Boksenberg and myself (first by Peterson and myself), have small rotation. Unpublished preliminary work on DB stars also suggests small rotation. The single magnetic stars, also, have slower rotation than suggested by conserving angular momentum during evolution. Apparently we need not expect substantial deviations from the classical $M-R$ relation to be caused by rotation, and it is probable that meridional circulation is also slow. See Greenstein et al. (1977).

\section{THE TEMPERATURE SCALE}

Given the improvements of knowledge of the absolute energy distribution represented by the work of Hayes, Latham, Oke, Schild and others, wide-range scans of white dwarfs have been particularly useful for comparison with models; although Strymgen photometry is very precise, it is subject to systematic differences between observers, for each photometer and filter set. The optical limited spectral coverage from scans resulted in two parameters being useful--the slope on the high-frequency side of the Paschen 1imit, and the drop beyond the Balmer Iimit in DA stars. Strbmgren photometry measures these parameters also, and both methods have been extensively used recently to give a large number of temperatures and gravities (with less certainty). About 200 DA stars have Strbmgren or multichannel photometry. Since the varlous models now give essentially similar predicted fluxes, the temperature scale seems well defined, except for the hottest stars. Limited by our atmosphere, one cannot expect colors to determine high temperatures well. In Greenstein (1979), I conclude that either from two multichannel colors $m_{2.12}-m_{1.44}$ (called $G-R$ ), and $m_{2.80}-m_{1.85}$ (called U-V), or from fiteting the.44tire multichannel energy distribution to a model gives the same result, and up to $30,000 \mathrm{~K}$ quite reliably. Examining $45 \mathrm{DA}$ stars in common to Shipman (1979) and myself, $\log \mathrm{T}_{\mathrm{S}}$ $\log T_{G}=+0.002 \pm 0.004$, with $\sigma= \pm 0.028$ for one determination. The temperatures range from $7000 \mathrm{~K}$ to $30,000 \mathrm{~K}$; I used Koester's $\log \mathrm{g}=8$ models, while Shipman used his own models. These LTE, blanketed models compare quite well, and also fit observations such as the MCSP data in Greenstein (1976b).

Others eventually must compare the results for helium stars; since I had no grid of model atmospheres for work on stars with helium atmospheres (1976a). Shipman (1979) gives data on 28 cooler, probably helium-rich stars, including magnetic and carbon types 6000 to $17,000 \mathrm{~K}$. Koester (1979) has supplied predicted colors on the baseline $\mathrm{m}_{2}, 08$ $m_{1.36}$ (called $G^{\prime}-R^{\prime}$ ), and $U-V$ which indicate that $D B$ stars showing He I reach $30,000 \mathrm{~K}$; He I lines disappear at my resolution below $12,000 \mathrm{~K}$. The numerous yellow DC stars have colors which form a natural extension of helium atmosphere colors to much lower temperatures. Here again, 
more models are needed. Koester has analyzed the multichannel data on helium degenerates and derives a lower mean $\log \mathrm{g}$ and mass (1979).

Metals appear in many helium-atmosphere stars in this cooler range, with varying intensities, and in GD 40 (Shipman, Greenstein, and Boksenberg 1977) Ca II is seen at $15,200 \mathrm{~K}$. A very interesting group of stars bluer than established $\mathrm{DB}^{\prime}$ 's have been found colorimetrically by $\mathrm{R}$. Green (in progress). They have strong He I lines; little is known about their proper motions or their luminosities; the line broadening is not sufficlent alone to establish by inspection whether they are DB's hotter than $30,000 \mathrm{~K}$, or hydrogen-poor cooler relatives of the sdo group with intermediate $\log g$ values. Some intermediate composition stars may exist, types $\mathrm{DBA}$ or $\mathrm{DAB}$; in addition to sdo stars with little He II, or little $H$, or with both.

In late stages of the evolution of helium-carbon cores, the helium degenerate core of minimum mass $\left(0.54 \mathrm{M}_{\ominus}\right.$, say) may grow, during shell burning, to larger masses elther in hellum- or hydrogen-envelope stars. A mass as low as $0.5 \mathrm{M}_{0}$ has an expected Einstein shift $24 \mathrm{~km} \mathrm{~s}$; at $0.7 \mathrm{M}_{\Theta}$ this is $40 \mathrm{~km} \mathrm{~s} \mathrm{e}^{2}$ and at $0.9 \mathrm{M}_{\Theta}, 65 \mathrm{~km} \mathrm{~s}^{-1}$. If the final mass depends on details of late evolutionary stages, it should be determinable from gravitational redshift measures, perhaps as well as from determinations of $\log \mathrm{g}$ (which ranges theoretically from 7.9 to 8.5 for the above mass range).

I have a private doubt concerning the nature of very high-temperature white dwarfs. It is of the greatest theoretical importance to know how hot a degenerate star can be, because of the still uncertain final evolutionary connection between either nuclei of planetary nebulae, horizontal branch, sdB, or hot sdo stars, and the hottest fully degenerate star. Many of the hottest known DA white dwarfs are in binary systems; Felge 24 is a much closer spectroscopic binary, with some interaction. One must wonder whether this is an accident or whether there is a causal connection. In PG 1413+015, Schmidt and Green ascribe $50,000 \mathrm{~K}$ temperature to their elght hour eclipsing binary containing a $\mathrm{dM}, 10^{11} \mathrm{~cm}$ from a white dwarf. Wray, Parsons, and Henize (1979) add another, unusual He II-rich object, HD 149499B, which they observed with the IUE. Its spectrum resembles some sdo's. They ascribe a temperature near $85,000 \mathrm{~K}_{14}$ At 2 arcseconds distance from a KOV primary, the separation is $9 \times 10^{14} \mathrm{~cm}$; the degenerate star subtends so small an angle that it would intercept geometrically only $10^{-13}$ of any stellar wind from the primary. At a mass-loss rate of $10^{-9} \mathrm{M}_{\theta} \mathrm{yr}^{-1}$, and neglecting any focussing or radiation-pressure effects on the capture rate, the white dwarf receives so small a_pass that, at the velocity of infall, this $m$ yields only $2 \times 10^{21}$ erg $\mathrm{s}^{-1}$. But the white-dwarf radiation may force more rapid mass loss, or magnetic-field funneling reduces the enormous dilution of the stellar wind. Possible mechanisms of heating in binaries need some thought. The spectrum of a hot model atmosphere differs greatly from that of an accretion disk, so that it is not likely that these high $T$ are only apparent. Table I gives a list of these hottest stars, with rough linear separations. The frequency of binaries is high, possibly even too high, for chance. 


\begin{tabular}{|c|c|c|c|c|c|c|}
\hline Object & Ref. & Type & $\mathrm{T}_{3}$ & Pair & $\begin{array}{c}\text { Dist. } \\
\mathrm{cm}\end{array}$ & Comments \\
\hline HD 149499B & 1 & DO & 85: & Vis., 2", KO & $10^{15}$ & $\begin{array}{l}\text { Might be He high-g } \\
\text { subdwarf. }\end{array}$ \\
\hline HZ 43 & 2 & DAwk & $60+$ & Vis., 3", dMe & $2 \times 10^{15}$ & EUV source; EG 98 . \\
\hline Feige 24 & 3 & DAwk & 60 & Spect., dMe & $10^{11}$ & $\begin{array}{l}\text { Interacting binary; } \\
\text { EG } 20 ; P=4 \text { days. }\end{array}$ \\
\hline G191B2B & - & DAwk & $50-60$ & Vis., sdK & $5 \times 10^{16}$ & EG 247 \\
\hline HZ 21 & 4 & DO & 48 & Single & $\cdots$ & $\mathrm{He} / \mathrm{H} \approx 10 ; \mathrm{EG} 86$. \\
\hline PG1413+015 & 5 & $\mathrm{DC}:$ & $>50$ & $\begin{array}{l}\text { Eclipsing } \\
\text { Binary }\end{array}$ & $10^{11}$ & $\begin{array}{l}\text { Late } M \text {, not seen. } \\
P=8 \text { hours. }\end{array}$ \\
\hline HZ 34 & 4 & $\mathrm{DAB}$ & 50 & Single & $\cdots$ & EG 93 \\
\hline GD 561 & - & DAvwk & 50 & Single & $\cdots$ & $\begin{array}{l}\text { Gr } 304 \text {. Motion small, } \\
\text { possible subdwarf. }\end{array}$ \\
\hline
\end{tabular}

The list contains all those known to Shipman or myself, $T>50,000$. Many dM+DA pairs known with low DA luminosity and T. Results ${ }^{6}$ of ANS photometry included.

1 - Wray, J. D., Parsons, S. B., and Henize, K. G. 1979, Ap. J. (Letters), in press. In this, IUE spectrum is atypical, showing high members of $\lambda 4686$ series.

2 - Auer, L. H., and Shipman, H. L. 1977, Ap. J. (Letters), 211, L103. Temperature depends on low $\mathrm{He} / \mathrm{H}$ ratio.

3 - Margon, B., Lampton, M., Bowyers, S., Stern, R., and Paresce, F. 1976, Ap. J. (Letters), 210, L79; Thorstensen, J. R., Charles, P. A., Margon, B., and Bowyer, S. 1978, Ap. J., 223, 260.

4 - Koester, D., Llebert, J., and Hege, E. K. 1979, Astr. Ap., 21, 163.

5 - Green, R. F., Richstone, D. O., and Schmidt, M. 1978, Ap. J., 224, 892.

6 - Wesselius, D. R., and Koester, D. 1979, Astr. Ap., 70, 745. 
New data on the ultraviolet spectra of white dwarfs gives new information that might have revolutionized our views. We have an unparalled opportunity in being able first to observe a number of such spectra with the IUE. This remarkable instrument gave Oke and myself (1979) a glimpse of the spectrophotometric properties of $3 \mathrm{DA}$ 's and one Do star, with Lya profiles of useful quality. The results were that the ultraviolet gave the same temperatures and (probably) surface gravities, when fitted to available model atmospheres, as had been obtained from the ground, using the multichannel spectrophotometer (Shipman 1979; Greenstein 1979). Oke will illustrate the DA spectra later in this conference. Table II compares the results at present available for a mixture of magnetic, hydrogen and helium atmosphere white dwarfs. The IUE temperature determinations must be viewed as preliminary, since the spectrophotometric stability is only moderate and a new error in calibration has been discovered, especlally for faint stars measured in the presence of a radiation background. A further problem was the absence of suitable model-atmosphere predicted fluxes for those stars containing helium. Nevertheless, these further results are indeed exciting; the observations by Oke and myself will appear soon in detail. A further fact should be emphasized, that no evidence for any ultraviolet metallic lines has been so far found in any DA star.

The hydrogen-atmosphere DA's, of which 8 have been observed, range in temperature from 7000 to above $60,000 \mathrm{~K}$; if all have similar radii, this corresponds to a range of 9.3 in bolometric magnitude. Comparison between ground-based and IUE temperatures is shown in Table II, with Individual DA stars 1isted; since this grid of models is the most complete, errors are small, and the systematic agreement excellent. Models giving ultraviolet fluxes are from Koester, D. H. Peterson, McGraw and Shipman or Shipman computer printouts. One ZZ Ceti varlable, G29-38 (EG 159), is plotted. Its temperature is $11,500 \mathrm{~K}$ from IUE, and 12,000 $\mathrm{K}$ from Shipman or Greenstein using the normal spectral region. Variability apparently does not affect the ultraviolet flux.

When we attempt to do the same fitting of the few helium stars, DB (GD 190) or DBM (GD 40, Shipman, Greenstein, and Boksenberg), and HZ 21 (DO; Koester, Liebert, and Hege 1978) we were in a much worse situation, lacking at the time a grid of helium models. Considering the coarseness of the grid, the accidental and systematic errors seem satisfactory, the worst error being $\mathrm{HZ} 21$. The most interesting case is Ross 640, a helium atmosphere (with some hydrogen), rich in metals, and far too cool to show He I. An analysis by P. Cottrell is in progress, by the method of spectrum synthesis. He used the helium-model atmosphere at $8800 \mathrm{~K}$ that Liebert (1977) had used to fit the optical region, with the result that what continuum is visible, given the strong metallic lines, provides, in fact, a good fit. Therefore, the UV determination of temperature agrees moderately well with the $(8500 \mathrm{~K})$ of Liebert (1977). We will return to this star when we discuss compositions. A single, weak carbon band degenerate, L1363-3 needs a hellum-model somewhat cooler than the least-squares blackbody given.

Data on two magnetic stars observed will be discussed by Greenstein and Oke (1979; in preparation) in detall. Feige 7, which shows both $\mathrm{H}$ 
COMPARISON OF EFFECTIVE TEMPERATURES DETERMINED FROM GROUND BASED AND SPACE OBSERVATIONS

\begin{tabular}{|c|c|c|c|c|c|}
\hline \multirow[b]{2}{*}{ Name } & \multirow[b]{2}{*}{$E G, G R$} & \multicolumn{3}{|c|}{$\log \mathrm{T}_{\text {eff }}$} & \multirow[b]{2}{*}{ Type } \\
\hline & & Shipman & JLG & IUE & \\
\hline \multicolumn{6}{|c|}{ DA, Hydrogen Atmospheres } \\
\hline $\begin{array}{l}\text { L870-2 } \\
\text { Feige } 24 \\
40 \text { Eri B } \\
\text { HZ } 43 \\
\text { Wolf } 1346 \\
+73^{\circ} 8031 \\
\text { G29-38 } \\
\text { G191B2B }\end{array}$ & $\begin{array}{r}11 \\
20 \\
33 \\
98 \\
139 \\
144 \\
159 \\
247\end{array}$ & $\begin{array}{l}3.86 \\
4.78 \\
4.23 \\
4.80 \\
4.33 \\
4.19 \\
4.08 \\
4.79\end{array}$ & $\begin{array}{c}3.86 \\
(4.78) \\
4.21 \\
\ldots . .3 \\
4.33 \\
4.20 \\
4.08 \\
4.75\end{array}$ & $\begin{array}{c}3.85 \\
<4.84 \\
4.22 \\
(4.78) \\
4.34 \\
4.18 \\
4.06 \\
4.78\end{array}$ & $\begin{array}{l}\text { DA } \\
\text { DA } \\
\text { DA } \\
\text { DAwk } \\
\text { DA } \\
\text { DA } \\
\text { DAV } \\
\text { DAwk }\end{array}$ \\
\hline \multicolumn{6}{|c|}{ Helium Atmospheres } \\
\hline $\begin{array}{l}\text { HZ } 21 \\
\text { Ross } 640 \\
\text { L1363-3 } \\
\text { GD } 190 \\
\text { GD } 40\end{array}$ & $\begin{array}{r}86 \\
119 \\
148 \\
193 \\
384\end{array}$ & $\begin{array}{l}(4.70)^{b} \\
3.93 \mathrm{H}^{c} \\
3.93 \\
(4.26)^{b} \\
4.15(\mathrm{SGB})\end{array}$ & $\begin{array}{l}4.68 \mathrm{KLH} \\
3.89 \mathrm{~L} \\
\ldots . \\
4.34 \\
\ldots\end{array}$ & $\begin{array}{l}4.60 \mathrm{~W} \\
3.94 \\
(3.98) \mathrm{BB} \\
4.36 \\
<4.18\end{array}$ & $\begin{array}{l}\text { DO } \\
\text { DFp } \\
\text { DC } 2^{\text {wk }} \\
\text { DB } \\
\text { DBM }\end{array}$ \\
\hline \multicolumn{6}{|c|}{ Magnetic } \\
\hline $\begin{array}{l}\text { Grw }+70^{\circ} 8247 \\
\text { Feige } 7\end{array}$ & $\begin{array}{l}129 \\
267\end{array}$ & $\begin{array}{r}4.14 \\
\ldots\end{array}$ & $\begin{array}{l}(4.13) \\
4.34(G B)\end{array}$ & $\begin{array}{l}4.18(\mathrm{BB}) \\
4.36(\mathrm{~W})\end{array}$ & $\begin{array}{l}\text { DXP } \\
\text { DABP }\end{array}$ \\
\hline
\end{tabular}

References: $\mathrm{BB}=$ blackbody; $\mathrm{GB}=$ Greenstein and Boksenberg, $\underline{\text { MNRAS }}$, 185, 823, 1978; SGB = Shipman, Greenstein, and Boksenberg, A. J., 82, 480, 1977; (w) =Wickramasinghe, Mem. R.A.S., 76, 129, 1972; L = Liebert, Astr. Ap., 60, 101, 1977; KLH - Koester, Liebert, and Hege, Astr. Ap., 21, 163, 1979; $W=$ Wesemael, unpublished model.

${ }^{a}$ Composite, red companion affects MCSP.

${ }^{b}$ Shipman Thesis.

CHammond Thesis. 
and He I Zeeman-resolved lines can be analyzed using Wickramasinghe's (1972) ${ }_{4}$ DB models, since, fortunately, he had allowed the $\mathrm{H} / \mathrm{He}$ ratio to be $10^{-4}$. As a result, although no Balmer jump is seen, a Lya line is, and his model gives a reasonable fit to line and continuum. In fact, the many Zeeman lines depress the optical region extensively, so that the temperature is equally poorly (or well) determined in both portions of the spectrum. For Grw $+70^{\circ} 8247$, the ultraviolet spectrum is so highly distorted that only a blackbody $f$ it is possible, at a temperature differing by 12 percent from that determined in the relatively smooth optical region (Greenstein 1979) from the color-color properties of that star. The most striking result, however, is the integrated low flux of thif star, which in the observed wavelengths covered is $3.3 \times 10^{-10}$ erg

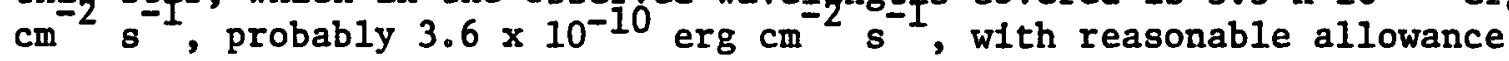
for the far infrared and ultraviolet. From an accurate parallax, the luminosity is $7.1 \times 10^{30} \mathrm{erg} \mathrm{s}^{-1}$; within the range of effective temperatures 10,000 to $16,000 \mathrm{~K}$, the radius varies from 0.0135 to $0.0053 R_{\theta}$; however, the IUE flux in the ultraviolet is not consistent with $T<$ $14,000 \mathrm{~K}$ (where there is a large ultraviolet excess). The minimum of the residuals, $1 . e .$, the "least squares" fit to a blackbody curve is at $16,000 \mathrm{~K}$ where the radius $1 \mathrm{~s} 0.054 \mathrm{R}_{0}$. This would be much the smallest known white dwarf radius, corresponding to $1.23 \mathrm{M}_{\theta}$ (for a helium-core, zero-temperature degenerate configuration). Even the lowest plausible temperature gives a mass $1.03 \mathrm{M}_{\theta^{\circ}}$. It would be important to obtain a parallax also for Feige 7, where the temperature is better determined. The magnetic, polarized white dwarf (DXP type) LP44-113 = G240-72 (Gr 372) had been noted to have a remarkably low luminosity $M_{v}=15.22$ by the USNO parallax observers (Dahn et al. 1976).

\section{BRIEF COMMENTS ON COMPOSITION}

Few or no hydrogen degenerates show metallic lines. The upper limits to the metal abundances in DA's are of little practical usefulness, and a spectrum that shows $\mathrm{H}$ and $\mathrm{Ca}$ II, like that of Ross 627 (EG 79), has been interpreted as a mixed helium-hydrogen atmosphere in which metals do appear. The usual breakdown of degenerate stars into DA or non-DA is actually a separation between nearly pure $\mathrm{H}$ or pure He (with some metals). It is to be hoped that a more physically meaningful spectral classification will soon be adopted. The He/H ratio in DA's is at most $10^{-3}$; in $\mathrm{HZ} 43$, rocket observations with a spectrometer devel-

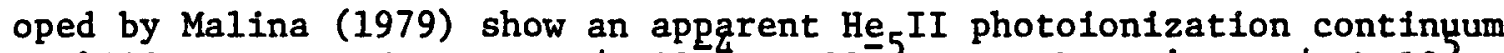
at $\lambda 228$ corresponding to $\mathrm{He} / \mathrm{H}^{-4}$ or $10^{-5}$. In the $\mathrm{DB}^{\prime} \mathrm{s}_{3} \mathrm{He} / \mathrm{H} \geq 10^{5}$, with some mixed $\mathrm{DBA}$ atmospheres near $\mathrm{He} / \mathrm{H}=3$ to $10 \times 10^{3}$. Cool $\mathrm{DB}^{\prime} \mathrm{s}$ show no He I lines, are classified as $\mathrm{DC}^{\prime} \mathrm{s}$. The transparency of helium occasionally results in the appearance of some metals. Several magnetic stars are mixed, DBA stars. In cool DBA's, H $\alpha$ has been detected, in one magnetic star, $\mathrm{CH}$. The "carbon" degenerates are a limited group of He-dominated atmospheres, with about the solar $\mathrm{C} / \mathrm{He}$ ratio, and no metals. The few DO stars are quite hot and have intermediate $\mathrm{He} / \mathrm{H}$ ratios; only one has been quantitatively analyzed. Very blue stars, far above the $30,000 \mathrm{~K}$ limit of DB stars have been found in Green's Palomar survey, many apparently of mixed composition, and will require further study. The sdo stars have a variety of $\mathrm{He} / \mathrm{H}$ and probably of metal-to-helium ratios. Recent non-LTE quantitative analysis by Simon et al. (1979) of a sdO in a binary system, HD 49798 gives $\mathrm{He} / \mathrm{H} \approx 1$. My 
own impression from Greenstein and Sargent (1974) 1s that some sdo's show essentially only $H$, some only He II and He $I$, and others $H$, He II and He I. Oke, in this conference, will show IUE observations of sdo's; some seem to be losing mass; some are very hellum rich. In their atmospheres the usual highly-lonized elements, C, N, Si, are seen.

But for this I confine myself to the evidence on metal abundances in degenerate stars with dominantly helium atmospheres. Advances in the theory of gravitational settling, diffusion, convection and accretion have been substantial; a review of the observations (see also, Vauclair, Vauclair, and Greenstein 1979) displays the variety of metal abundances found. In most cases, data is taken from the published results, using the adopted temperatures, except for G165-7, for which I am Indebted to $R$. Wehrse (in press), and Ross 640, where P. Cottrell is analyzing my IUE data by spectral synthesis. Table III shows the results for stars hotter than vMa2, and includes upper limits for a DC. Note that the spectral classifications used must be viewed as on an outmoded system, and should soon be replaced by a more rational one which separates helium from hydrogen stars, gives a temperature indicator, and then indicates the presence of metals. The star GD 40, called DBM, has both He I and $\mathrm{Ca}$ II lines; it shows $\mathrm{Mg}$ II $\lambda 2800$ in its IUE spectrum. G111-54 is slightly cooler, and really very similar, but at present can only be called DF. In the table, $T_{3}$ is the effective temperature in $10^{3} \mathrm{~K}$, and all abundances are $\log \mathrm{n}(\mathrm{M}) / \mathrm{n}(\mathrm{He}) \equiv-\mathrm{N}$. In the abundance column $P$ means present, but no quantitative results yet available. In the fifth column $W(K)$ gives an approximate strength of $H$ and $K$, if blended, or $\mathrm{K}$ alone. Note that if any general trend exists, it is that the cooler strong-lined stars have, in fact, relatively lower abundances of metals to helium than do the few hot stars that show $\mathrm{Ca}$ II. If the $\mathrm{Fe} /$ Ca ratio is preserved, then the hot stars should have very strong ultraviolet resonance multiplets of Fe II, worth searching for with the IUE. The solar abundances, at the bottom, are standard values, $1 \circ \mathrm{g} \mathrm{M} / \mathrm{H}$; were all hydrogen processed into $\mathrm{He}$, then $\log (\mathrm{M} / \mathrm{H}+\mathrm{He})$ should be these values plus 0.5. Even the most metal-rich star so far analyzed is strongly metal deficient, except possibly GD 401. If that star fits the same model as Ross 640 , then if $\mathrm{N} \propto \mathrm{W}^{1 / 2}$, then $-\mathrm{N}_{1}=7.8$, 1 .e., still metaldeficient. Most upper limits to metal abundances derived from the absence of lines in the optical regions are $10^{-10}$ to $10^{-11}$. The data cannot establish any preferential abundance enhancement or deficiency as yet, although $\mathrm{Ca}$ II when present seems to have the lowest deficiencies. The most promising avenue for future exploration of this question with the IUE or Space Telescope, is the Fe II or Mg II ratio to Ca II.

Ross 640 is a particularly well-studied object in which Liebert (1977) found a weak $\mathrm{H} \alpha$, corresponding to a quite low hydrogen abundance. Cottrell's synthetic spectra were based on Liebert's model, for which we are grateful. The IUE spectrum is well represented, except that the core and wings of $\lambda 2800$ of $\mathrm{Mg}$ II are not well-fitted simultaneously, abundance differences by a factor of three being required. The multiplets of $\mathrm{Fe}$ II used in synthesis went up to $1 \mathrm{eV}$ excitation (UVMT, $\$ 1-6,32-36,60-64)$ and give $n(\mathrm{Fe}) / \mathrm{n}(\mathrm{He})=2 \times 10^{-9}$, while Liebert had set an upper limit from the optical region at $<2.5 \times 10^{-9}$; similarly, the ultraviolet gives $\mathrm{n}(\mathrm{Si}) / \mathrm{n}(\mathrm{He})=3 \times 10^{-8}$, while Liebert's value was $<10^{-7}$. The most significant datum is the $\mathrm{Mg} / \mathrm{Fe}$ ratio which seems well 
SUMMARY METAL ABUNDANCES IN HELIUM ATMOSPHERE DEGENERATES

\begin{tabular}{|c|c|c|c|c|c|c|c|c|c|}
\hline Star & $\begin{array}{l}\mathrm{EG} \\
\mathrm{Gr}\end{array}$ & Type & $T_{3}$ & $\begin{array}{l}\text { O } \\
W(A) \\
\mathrm{Ca} \text { II }\end{array}$ & $\begin{array}{r}-N_{1} \\
M g \\
\end{array}$ & $\mathrm{SI}$ & $\begin{array}{r}\mathrm{n}(\mathrm{M} \\
\mathrm{Ca} \\
\end{array}$ & $\begin{array}{c}\left.1_{1}\right) / n( \\
F e\end{array}$ & Ref. \\
\hline $\begin{array}{l}\text { GD } 40 \\
\text { G111-54 } \\
\text { GD } 401 \\
\text { Ross } 640 \\
\text { L745-46A } \\
\text { G165-7 } \\
\text { Ross } 627 \\
\text { Stein 2051B } \\
\text { G139-13 } \\
\text { v Ma2 } \\
\text { Solar }\end{array}$ & $\begin{array}{r}384 \\
346 \\
451 \\
119 \\
54 \\
391 \\
79 \\
180 \\
494 \\
5\end{array}$ & $\begin{array}{l}\text { DBM } \\
\text { DF } \\
\text { DFp } \\
\text { DFp } \\
\text { DF } \\
\text { DG } \\
\text { DA, F } \\
\text { DC } \\
\text { DG } \\
\text { DG } \\
. .\end{array}$ & $\begin{array}{l}15 \\
12 \\
9.5 \\
8.8 \\
7.8 \\
7.5 \\
7.2 \\
7.0 \\
6.6 \\
5.5 \\
. . .\end{array}$ & $\begin{array}{r}4 \mathrm{~B} \\
18 \\
170 \\
36 \\
7 \\
\mathrm{BL} \\
1 \\
<1 \\
105 \\
\mathrm{BL} \\
\ldots .\end{array}$ & $\begin{array}{r}\ldots \\
\ldots \\
\ldots .4 \\
6.4 \\
4.4 \\
4.9 \\
>10 \\
\ddot{10} \\
9.4 \\
3.7\end{array}$ & $\begin{array}{l}\ldots \\
\cdots \\
\ddot{6} .7 \\
>9 \\
4.9 \\
\cdots \\
\cdots \\
\ddot{>} \\
3.7\end{array}$ & $\begin{array}{c}7.5 \\
P \\
>7.0 \\
8.3 \\
10.4 \\
6.6 \\
10 \\
>10 \\
P \\
10.7 \\
4.9\end{array}$ & $\begin{array}{r}\ldots . \\
\ldots \\
\ddot{8} .5 \\
>9.4 \\
5.5 \\
>10 \\
\ldots 10 \\
9.7 \\
4.3\end{array}$ & $\begin{array}{l}\text { SGB } \\
\text { G1 } \\
\text { G2 } \\
\text { G3, Cott. } \\
\text { Weg., G3 } \\
\text { WL } \\
\text { Ship. } \\
\text { L } \\
\text { G1 } \\
\text { Weg. } \\
\text { - }\end{array}$ \\
\hline
\end{tabular}

\section{Notes:}

In this Table uncertainties arise from the comparison of metal abundances with He rather than $\mathrm{H}$ and in the solar abundances adopted.

$\mathrm{B}: \mathrm{K}=3, \mathrm{H}$ blended $\mathrm{H} \varepsilon ; \mathrm{BL}$ Ca II badly blended, strong;

G1: Greenstein unpublished; G111-54 observed w1th Boksenberg. G139-13 must have high $\mathrm{Ca}$, in range 7 to 10 .

G2: Greenstein, J. L. 1976, Ap. J., 210, 524. Abundance estimate based on comparison with Ross 640.

G3: Greenstein, J. L. 1960, Stellar Astronomy, ed. J. L. Greenstein (Chicago: University of Chicago Press).

L: Liebert, J. 1976, Ap. J., 210, 715.

Ship.: Shipman, H. L. 1972, Ap. J., 177, 723. Estimate only; since he used H-atmosphere, < 10 .

SGB : Shipman, H. L., Greenstein, J. I, and Boksenberg, A. 1977, A. J., 82,7 .

Weg.: Wegner, G. 1972, Ap. J., 172, 451.

WL:' Wehrse, R., and Liebert, J. unpublished. Their conclusion, "metals $\approx 4 \times 10^{-5}$ solax, except for $\mathrm{Ca}$ down by factor three." 
above the solar value. Attempts to observe $v$ Ma2 with the IUE haye falled, so far, presumably because the near ultraviolet is very strongly depressed by line blanketing.

This work has been supported in part by a grant from the National Science Foundation (AST 77-09191) and from the National Aeronautics and Space Administration, to myself and J. B. Oke, NSG 5243.

\section{REFERENCES}

Dahn, C. C., Harrington, R. S., Riepe, B. Y., Christy, J. W., Guetter, H. H., Behall, A. L., Walker, R. L., Hewitt, A. V., and Ables,

H. D. 1976, U. S. Naval Obs., 24, Part 3.

Green, R. 1977, Thesis, California Institute of Technology. Greenstein, J. L. 1976a, Ap. J., 210, 524 . - $1976 \mathrm{~b}, \mathrm{~A} . \mathrm{J} ., 8 \overline{1}, 323$. - 1979, Ap. J., in press.

Greenstein, J. L., Boksenberg, A., Carswell, R., Shortridge, K. 1977, Ap. J., 212, 186.

Greenstein, J.L., and Oke, J. B. 1979, Ap. J. (Letters), 229, L141. Greenstein, J. L., and Sargent, A. I. 1974, Ap. J. Supp1., 28, 157. Koester, D. 1978,1979, computer printouts and these Proceedings. Koester, D., Liebert, J., and Hege, E. K. 1979, Astr. Ap., 71, 163. Koester, D., Schulz, H., and Weldemann, V. 1979, Astr. Ap., In press. Llebert, J. 1977, Astr. Ap., 60, 101.

Malina, R. 1979, Thesis, University of California, Berkeley, and preprint. See also, Bowyer, S., these Proceedings.

McGraw, J. T. 1977, Thesis, University of Texas, and these Proceedings. McGraw, J. T., and Shipman, H. I. 1978, computer printout.

Shipman, H. L. 1978, computer printouts. - 1979, Ap. J., 228, 240.

ShIpman, H. L., Greenstein, J. I., and Boksenberg, A. 1977, A. J., 82,480 .

Simon, K. P., Gruschinske, J. Hamann, W. R., Hunger, K., and Kudritski, R. P. 1979, preprint; also Kudritski, R. P., and Simon, K. P. 1978, Astr. Ap., 70, 653.

Vauclair, G., Vauclair, S., and Greenste1n, J. L. 1979, Astr. Ap., in press.

Wehrse, R., and Liebert, J. 1979, private communication. Wickramasinghe, D. T. 1972, Mem. R.A.S., 76, 129.

Wray, J. D., Parsons, S. B., and Henize, K. 1979, preprint, Ap. J. (Letters), in press. 